\title{
Expression of the Protein Product Genenm23 in Squamous Carcinoma of the Tongue
}

\author{
Harmas Yazid Yusuf ${ }^{1}$, Bethy S Hernowo ${ }^{2}$, Nani Murniati ${ }^{3}$ \\ ${ }^{1}$ Department of Oral and Maxillofacial Surgery Faculty of Dentistry, Padjadjaran University, Sumedang, Indonesia \\ ${ }^{2}$ Department of Pathology, Faculty of Medicine, Padjadjaran University, Sumedang, Indonesia \\ ${ }^{3}$ Department of Oral Biology, Faculty of Dentistry, Padjadjaran University, Sumedang, Indonesia
}

\begin{abstract}
Tongue squamous cell carcinoma, a relatively frequent malignancy in oral cavity, has a great tendency to metastasize to regional lymph nodes that worsen the prognosis of the disease. The antimetastasisgene NM23, encodes a 17 KD cytoplasmic and nuclear protein, has shown controversial behaviour as metastatic suppressor in human cancer. A study using immunohistochemical staining method with LSAB technique was conducted on tongue squamous cell carcinoma paraffin block specimens to show the relationship between significance of the expression $\mathrm{OF} N \mathrm{NM23}$ protein product gene as a marker for cancer metastasize, with cancer grading and lymph nodes metastasis. The result showed that there was in inverse relationship between expression of NM23 protein and grading as well as lymph nodes metastases, supporting the role of NM23 as metastasis suppressor factor that may be useful for predicting tongue squamous cell carcinoma metastasis.
\end{abstract}

Keywords: NM23, tongue, carcinoma

\section{Introduction}

Metastasis to the lymph nodes is the most important factor in determining the prognosis of carcinoma sufferers. Aloe carcinoma has the ability to metastasize to regional lymph nodes greater than carcinomas in other parts of the oral cavity, due to the large amount of lymphatic webbing and its anatomical structure, with an incidence between $15-75 \%$ depending on the expansion of the primary lesion ${ }^{1}$. The NM23 gene was first identified by Steeg et al as a cancer metastatic suppressor gene in murine melanoma cell lines ${ }^{2-4}$. At the moment, five NM23 genes have been isolated in humans, namely NM23-H1 to NM23-H5. ${ }^{4} \mathrm{NM} 23-\mathrm{H} 1$ and NM23-H2 genes that are $88 \%$ homologous are found on chromosome 17q21-22.3, while NM23-H3 and NM23-H4 genes which are not homologous are found on chromosomes $16 q 13$ and 16p13.3 and the NM23-H5 gene on chromosome $5 q 23-31 .^{4}$ The protein product of the NM23 gene is known by various names such as AWD (abnormal wing disc) found in the Drosophila fly, factor I (differentiator inhibitor) and $\mathrm{PuF}$ (transcription factor in c-mycgene) factors. This product belongs to a large family of proteins with a molecular weight of $16-20 \mathrm{kD}$ which has NDPK (nucleoside diphosphate kinase) activity, which is an enzyme found in almost all parts of the cell that functions to catalyse the phosphorylation of 5 'triphosphate necleoside (NTP) to 5 'diphosphate nucleoside through a back and forth mechanism (pingpong mechanism) through the mediation of highenergy oxidative phosphorylation enzymes. ${ }^{4}$ The NDPK enzyme plays a role in the signal transduction process from the cell membrane to the cell nucleus through the activation pathways of proteins $\mathrm{G}$ and P21 and functions in cell division and maintains cell shape. ${ }^{4}$

The protein product of the NM23 gene has implications in cell proliferation and differentiation and the occurrence of cancer. ${ }^{4}$ The function of the presence of the NM23 gene and its protein product in human cancer is still controversial.
Some reports show a link between the high expression of the NM23 gene and its protein products and the low potential of cancer metastases, while other reports show the opposite results. It is therefore important to determine the protein expression of the NM23 gene product as a sign of cancer metastasis, especially of squamous cell carcinoma of the tongue. ${ }^{2,3}$

The purpose of this study was to observe the pattern of NM23 gene product protein expression in tissue squamous cell carcinoma of the tongue (KSSL) in paraffin blocks by immunohistochemical strokes and its relation to gradations and metastases to regional lymph nodes of the head and neck

\section{Materials and Method}

A total of 41 tongue carcinoma paraffin block networks obtained from the Department Pathology Faculty of Medicine, University of Padjadjaran / Dr. Hasan SadikinHospital Bandung were included in this study, through a purposive sampling technique based on the eligibility criteria of paraffin block networks, namely nondamaged paraffin blocks and KSSL networks are still sufficient to be able to at least three new preparations have been made as well as a complete reference form to the Department Pathology. The paraffin block is then made a new preparation, one preparation for hematoxylin eosin smear, that is for reassessment of histopathological diagnosis, another preparation for immunohistochemical smear of the protein product of the NM23 gene product and one preparation for negative control. As a negative control primary antibody substitution with phosphate buffered saline / PBS was used, while the positive control was breast carcinoma tissue. Positive control in the form of known tissue will express the antigen if it is processed by the same method as the cell to be examined, whereas negative control by replacing primary antibodies with diluted antibodies or with antibodies that are clearly incompatible with the 
antigens to be examined 5 Age, sex, gradation and metastatic data for head and neck $(\mathrm{N})$ regional lymph nodes were obtained from secondary medical record data. Gradient squamous cell carcinoma of the tongue is divided into gradations I (well differentiated), gradations II (moderately differentiated) and gradations III (poorly differentiated) using a classification of histological differentiation from the World Health Organization (WHO). ${ }^{6}$

The expression of NM23 protein gene in KSSL tissue was evaluated by the immunohistochemical smear by the labelled streptavidine biotin plus (LSAB ${ }^{\circ}$ plus) method (Dako Corp. Denmark). The paraffin block for each sample is cut to a thickness of 4 microns and placed on silane slides, then deparafinized in xylene solution. Retrieval antigen procedure is carried out by heating the preparation in a microwave oven at $60 \mathrm{C}$ for 20 minutes in a $4 \% \mathrm{AlCl} 3$ buffer medium. To eliminate endogenous peroxidase, the preparation is immersed in $3 \% \mathrm{H} 2 \mathrm{O} 2$ for 5 minutes. The NM23-H1 monoclonal primary antibody (NM301 Santa Cruz Biotech. Inc., CA, USA) was diluted 1:30 and the preparation was incubated at $37 \mathrm{C}$ for 60 minutes. The preparation was incubated in biotin labelled swine anti rabbit Ig antibody with a dilution of 1: 300 for 45 minutes at room temperature. In the next step, the incubation was incubated in streptavidin with a dilution of 1: 500 for 30 minutes at room temperature and finally applied $0.05 \%$ DAB (3.3 'diaminobenzidine tetrachloride) with a counter staining Hematoxylin Mayer. Evaluation of NM23 protein expression in tongue carcinoma tissue cells was carried out with the following semi-quantitative assessment, namely negative expression if $0-5 \%$ of KSSL immunopositive tissue cells, and positive expression if $>5 \%$ of immunopositive KSSL cells. Observation was carried out under a light microscope with $200 \mathrm{x}$ magnification of all KSSL preparations to get 500 cells. The data obtained were statistically analysed by Chi square test with $\mathrm{p}<0.01$.

\section{Result}

The protein expression of the NM23 gene product appears brownish in cytoplasmic cells and a portion of the nucleus cells in the tongue carcinoma tissue (Figure 1).

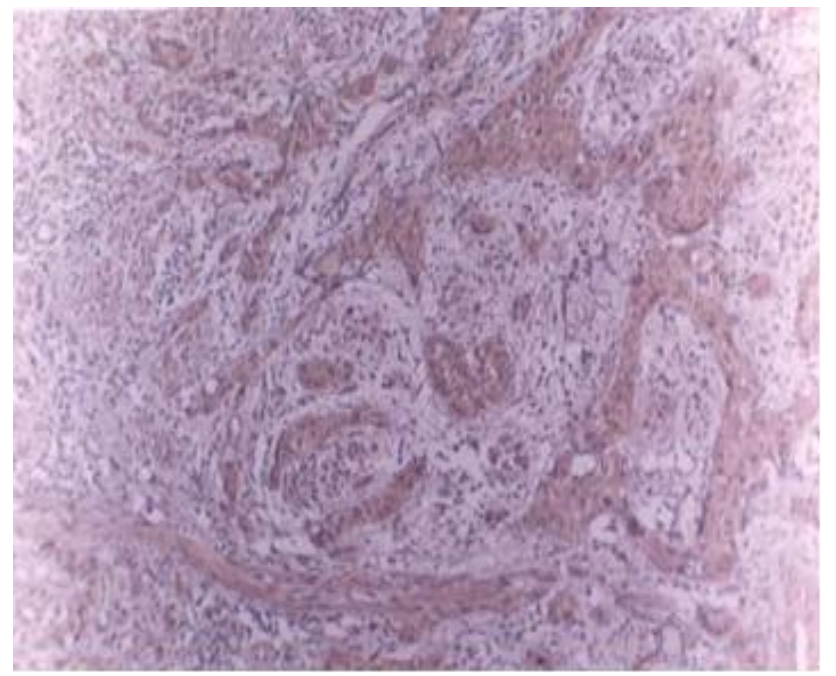

(A)

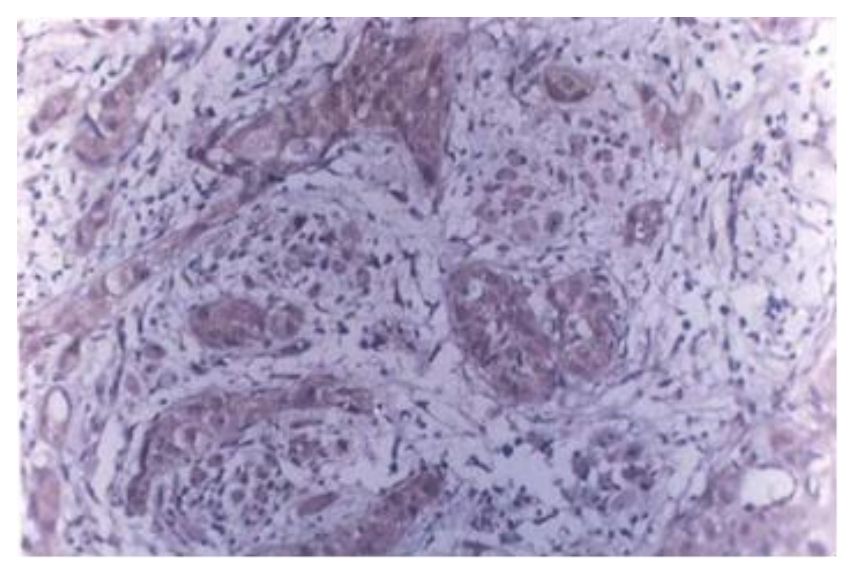

(B)

Figure 1: Immunopositivity of NM23 gene product protein expression in tongue carcinoma

a) Carcinoma cells show strong immunopositivity (brown color) to the NM23 protein (100x magnification)

b) Strong immunopositivity (brown color) to NM23 protein, especially in cytoplasm and carcinoma cell nuclei $(200 \mathrm{x}$ magnification)

The percentage of protein expression of NM23 gene products in tongue carcinoma was more dominant in negative NM23 expression than the percentage of positive NM23 expression (Table 1).

Table 1: Expression of NM23 gene product proteins in tongue carcinoma, cancer gradations and metastases to head and neck regional lymph nodes

\begin{tabular}{|c|c|c|c|c|c|c|}
\hline Expression & \multicolumn{2}{|c|}{ Frequency } & \multicolumn{2}{|c|}{ Gradation } & \multicolumn{2}{c|}{$\begin{array}{c}\text { Metastasis to regional } \\
\text { head and neck KGB (N) }\end{array}$} \\
\hline NM23 & $(\%)$ & I & II & III & Positive & Negative \\
\hline Positive & $15(34,6)$ & 12 & 3 & 0 & 2 & 13 \\
\hline Negative & $26(65,4)$ & 3 & 11 & 12 & 19 & 7 \\
\hline & $41(100)$ & 15 & 14 & 12 & 21 & 20 \\
\hline
\end{tabular}

The positive NM23 gene protein expression was dominant in Grade I, in 12 cases (80\%) compared to Grade II in 3 cases (20\%) and was not found in Grade III. The negative NM23 protein expression was mainly found in gradations II and III in 11 cases $(42 \%)$ and 12 cases $(46 \%)$, whereas in gradation I only 3 cases $(11 \%)$.

Head and neck regional lymph node positivity in KSSL was found in 21 cases $(51.3 \%)$, ie 2 cases $(9 \%)$ in positive NM23 gene protein expression and 19 cases $(91 \%)$ in negative NM23 gene protein expression (Table 1).

Chi squared test showed a significant relationship between protein expression of NM23 gene products in tongue carcinoma with cancer gradation and metastasis to regional lymph nodes $(\mathrm{p}<0.01)$. It can be said that the decrease in protein expression of NM23 gene products in tongue carcinoma has been empirically proven to increase or worsen cancer gradations and increase the frequency of metastases to regional head and neck lymph nodes. Age and sex data were not significantly related to NM23 protein expression. 


\section{Discussion}

The development of cancer and its progression to a metastatic phenotype are related to a series of genetic events involving the activation of cellular oncogenes and inactivation of cancer suppressing genes. The NM23 gene is a gene that shows the suppression activity of cancer metastases and its expression shows an inverse correlation with the metastatic potential in a number of human cancers ${ }^{7}$. The role of the complex NM23 gene in the metastasis process is marked by the diversity and different results from several studies on human cancer. The protein product of the NM23 gene often experiences increased expression in primary cancer when compared to the normal surrounding tissue $^{8,9}$, which is thought to be caused by activation in the early stages of carcinogenesis because immortalization of cancer cells will induce an increase in NM23 gene expression ${ }^{4}$. By continuing the process or stages of carcinogenesis, there will be a decrease in NM23 gene expression. ${ }^{4}$ The anti-metastatic role of the NM23 gene is due to the dysregulation of the process of apoptosis and suppression of MDR gene function ${ }^{1,10}$ The NM23 protein Affects the activation of protein G, inhibits myeloid differentiation, transduction of serine phosphorylation signals and regulation of transcription of other genes including the myc gene. ${ }^{10}$

This study shows that in the protein group the NM23 gene product with positive criteria, generally showed a relatively better cancer gradation and had a lower frequency of metastasis to the head and neck regional lymph nodes, compared to the negative NM23 protein criteria group.

It can be concluded that the NM23 gene and its protein product are strongly suspected to be a metastatic suppressor so that it can be used to predict metastasis and prognosis in squamous cell carcinoma of the tongue. The biological function and role of the NM23 gene mechanism have not been fully revealed, so that further research is needed regarding the mutation of the NM23 gene through gene amplification and DNA strand analysis to better understand the mechanism of oral carcinogenesis.

\section{References}

[1] Xin Xie, OPF Clause, De Angelis P, Boysen M. The prognostic value of spontaneous apoptosis, bax, bcl2 and p53 in oral squamous cell carcinoma of the tongue. Cancer 1999; 86: 913-20.

[2] De La Rosa A, Williams R, Steeg PS. Nm23 / nucleoside diphosphate kinase: toward a structural and biochemical understanding of its biological functions. Bioessays 1995; 17: 53-62.

[3] Lo Muzio L, Mignogna MD, Pannone G, Staibano S, Procaccini M, Serpico R, et al. The NM23 gene and its expression in oral squamous cell carcinoma. Oncology Reports 1999; 6: 747-51.

[4] Lombardi D, Lacombe M, Paggi MG. Nm23: Unraveling its biological function in cell differentiation. J Cell Physiol. 2000; 182: 144-9.

[5] Cote JR, Taylor CR. Immunohistochemistry and related marking technique. In Damjanov I, Linder J. Anderson's pathology. 10th ed. CV Mosby. St. Louis.136-66.
[6] Wahi PN, Cohen B, Luthra UK, Torloni, UK. Histological typing on oral and oropharyngeal tumors. In Wahi PN. International histological classification of tumors. Geneva 1971: 17-18.

[7] Lee CS, Redshaw A, Boag GB. Nm23-H1 protein immunoreactivity in laryngeal carcinoma. Cancer 1996; 77: 2246-2250.

[8] Lacombe MI, Sastre-Garau X, Lascu I, Wallet V, Thiery JP, Veron M. Overexpression of nucleoside diphosphate kinase (nm23) in solid tumors Eur J Cancer 1991; 27: 1302-7.

[9] Martinez JA, Prevot S, Nordlinger B, Nguyen TMA, Lacarriere $\mathrm{Y}$, Munier A, et al. Overexpression of NM23-H1 and NM23-H2 genes in colorectal carcinomas and loss of NM23-H1 expression in advanced tumor stages. Gut 1995; 37: 712-20.

[10] Charpin C, Bouvier C, Garcia S, Martini F, Andrac L, Lavaut MN, llasia C. Automated and quantitative immunocytochemical assays of nm23 / NDPK protein in breast carcinomas. Int J Cancer 1997; 74: 416-20. 